\title{
Tesis doctorals 2012-2017
}

\section{Doctorand/a: Apud Peláez, Ismael}

Títol: Science, Medicine, Spirituality and Ayahuasca in Catalonia.

Understanding ritual healing in the treatment of addictions from an interdisciplinary perspective

Any: 24/11/2017 | Universitat: URV

Direcció: Romaní Alfonso, Oriol; Czachesz, István

CCUC:

Doctorand/a: Carrillo Rosero, Ana María

Títol: Al cuidado del soberano. Des/orden, cuidado y vasallaje. Real

Audiencia de Quito 1780-1807

Any: 26/09/2017 | Universitat: URV

Direcció: Kingman Garcés, Eduardo; Comelles Esteban, Josep M.

CCUC:

Doctorand/a: Riccò, Isabella

Títol: En busca de un «nuevo mundo mágico». De la medicina popular a las terapias New Age en un Occidente desencantado

Any: 25/09/2017 | Universitat: URV

Direcció: Comelles Esteban, Josep María

CCUC:

Doctorand/a: Carceller Maicas, Natàlia Lledó

Títol: Por mímism@ saldré adelante. Percepciones, representaciones y prácticas en torno a los malestares emocionales en adolescentes y jóvenes Any: 22/09/2017 | Universitat: URV

Direcció: Martínez Hernaez, Angel

CCUC:

Doctorand/a: Tarifa Ortiz, Rafael

Títol: La Cooperativa Integral Catalana: una plataforma para una red de redes

Any: 20/09/2017 | Universitat: URV

Direcció: Vallverdú Vallverdú, Jaume; Pujadas Muñoz, Joan Josep CCUC: 
Doctorand/a: Bedón Cruz, Erika Natalia

Títol: Entre el poder y la gracia. Gobierno de poblaciones en espacios de acogida religiosos para personas marginales

Any: 13/09/2017 | Universitat: URV

Direcció: Kingman Garcés, Eduardo; Pujadas Muñoz, Joan Josep

CCUC:

Doctorand/a: Offenhenden, Maria

Títol: «Si hay que romperse una, se rompe». El Trabajo del hogar y la reproducción social estratificada

Any: 25/07/2017 | Universitat: URV

Direcció: Bodoque Puerta, Yolanda; Soronellas Masdeu, Montserrat

CCUC:

Doctorand/a: Alonso Rey, Natalia

Títol: Unas cuantas cosas. Objetos biográficos y experiencias migratorias

Any: 13/07/2017 | Universitat: URV

Direcció: Roca Girona, Jordi; Soronellas Masdeu, Montserrat

CCUC:

Doctorand/a: Espallargas Castro, José Luis

Títol: Screening Suburbia-Relaciones sociales, comportamientos y construcción de la identidad en las urbanizaciones residenciales. Una etnografía del espacio suburbano en la provincia de Tarragona Any: 26/01/2017 | Universitat: URV

Direcció: Maza Gutiérrez, Gaspar

CCUC: <http://www.tdx.cat/handle/10803/401581>

TDX: <http://hdl.handle.net/10803/401581>

Doctorand/a: Egbe Egbe, Manfred

Títol: Sub-Sahara African Immigrants in the 'Land of Plenty': Economic

Crisis, Food Insecurity and Hunger in Tarragona and Lleida

Any: 13/12/2016 | Universitat: URV

Direcció: Comelles Esteban, Josep M.; Gracia Arnaiz, Mabel

CCUC: <http://www.tdx.cat/handle/10803/401560>

TDX: <http://hdl.handle.net/10803/401560> 
Doctorand/a: Butnaru, Iulian

Títol: Naturaleza y alcance del símbolo

Any: 25/11/2016 | Universitat: URV

Direcció: Roda Aixendrí, Robert

CCUC:

TDX: <https://www.educacion.gob.es/teseo/mostrarRef.do?ref=1381185>

Doctorand/a: Guardi, Iolanda

Títol: Tendenze della letteratura algerina in lingua araba 1970-1980:

Identità nazionale, cittadinanza e condizione della donna

Any: 4/11/2016 | Universitat: URV

Direcció: Biglia, Barbara; Roca Girona, Jordi

CCUC:

Doctorand/a: Cardona Rodas, Hilderman

Títol: Iconografías médicas. Dermatología clínica en Colombia y España durante la segunda mitad del siglo XIX

Any: 13/09/2016 | Universitat: URV

Direcció: Comelles Esteban, Josep M.

CCUC: <http://www.tdx.cat/handle/10803/399229>

TDX: <http //hdl.handle.net/10803/399229>

Doctorand/a: Llort Suárez, Antoniu

Títol: «El pájaro está en el nido» Cocaína, cultura y salud: Más allá del modelo de adicción. Una etnografía del consumo de cocaína en Reus

Any: 14/03/2016 | Universitat: URV

Direcció: Romaní Alfonso, Oriol

CCUC:

Doctorand/a: Olaso Ruiz, Maria Julieta

Títol: «Me gusta cuando callas...» Análisis de los sistemas represivos de

Argentina y España y sus huellas a partir de la (re)construcción de la memoria democrática

Any: 09/02/2016 | Universitat: URV

Direcció: Vallverdú Vallverdú, Jaume; Roca Girona, Jordi

CCUC:

TDX: <http://hdl.handle.net/10803/399305>

Publicació: Julieta Olaso (2016): La represión y las luchas por la memoria en Argentina y España. Editorial Catarata. 288 páginas. ISBN: 978-84-9097241-0 
Doctorand/a: Olivella Quintana, Maria

Títol: Explorando las (im)posibilidades de una ley interseccional sobre violencias de género en el Estado español

Any: 08/02/2016 | Universitat: URV

Direcció: Roca Girona, Jordi; Biglia, Barbara

CCUC:

TDX: <http://hdl.handle.net/10803/399297>

Doctorand/a: Cover, Ilaria

Títol: Open Walls: The Experience of Psychiatric Institutionalization through Egyptian Women's Drawings

Any: 08/02/2016 | Universitat: URV

Direcció: Martinez Hernaez, Angel; Digiacomo, Susan M.

CCUC: <http://www.tdx.cat/handle/10803/380033>

TDX: <http://hdl.handle.net/10803/380033>

Doctorand/a: Arenas Paños, Miguel

Títol: Infarto de miocardio, infarto social. La experiencia de las

restricciones en un programa de rehabilitación cardiaca

Any: 08/02/2016 | Universitat: URV

Direcció: Gracia Arnaiz, Maria Isabel

CCUC: <http://www.tdx.cat/handle/10803/385860>

TDX: <http $/ /$ hdl.handle.net/10803/385860>

Doctorand/a: Yzusqui, Roxana

Títol: Hacia la protección internacional de la identidad cultural de los migrantes

Any: 03/02/2016 | Universitat: URV

Direcció: Castellà, Santiago J; Roca Girona, Jordi

CCUC:

TDX: <https://www.educacion.gob.es/teseo/mostrarRef.

do? ref $=1230462>$

Doctorand/a: Villa Camarma, Elvira

Títol: «Te maldecirán todas las naciones»: Prostitución, políticas públicas y biomedicina en los albores del siglo XxI

Any: 26/01/2016 | Universitat: URV

Direcció: Comelles Esteban, Josep M.

CCUC:

TDX: <https://www,educacion.gob.es/teseo/mostrarRef.do?ref=1251522> 
Doctorand/a: Maldonado Simó, Maria Luisa

Títol: Problematitzar el cos: repensar l'obesitat més enllà de la malaltia

Any: 21/12/2015 | Universitat: URV

Direcció: Gracia Arnaiz, María Isabel

CCUC: <http://www.tdx.cat/handle/10803/397780>

TDX: <http://hdl.handle.net/10803/397780>

Doctorand/a: Almagro Lorca, Maria Jesús

Títol: Proceso de socialización enfermera y construcción social de la profesión

Any: 10/12/2015 | Universitat: URV

Direcció: Roca Girona, Jordi

CCUC: <http://ccuc,cbuc,cat/ record $=$ b6739840 S23* cat >

TDX: <http://hdl.handle.net/10803/378644>

Doctorand/a: González Márquez, Miguel

Títol: Transformaciones urbanas en ciudades costeras e intermedias en el contexto Mediterráneo-Sur-Atlántico español

Any: 29/10/2015 | Universitat: URV

Direcció: Pujadas Muñoz, Joan Josep

CCUC: <http $: / /$ ccuc,cbuc,cat/ record $=$ b6651694 S23* cat >

TDX: <http://hdl.handle.net/10803/348559>

Doctorand/a: Piñones Rivera, Carlos

Títol: La mala hora. Articulaciones en el pluralismo médico de agricultores precordilleranos aymaras chilenos.

Any: 14/09/2015 | Universitat: URV

Direcció: Martínez Hernáez, Ángel; Ramírez Hita, Susana

CCUC: <http://ccuc.cbuc,cat/ record=b6617189 S23*cat>

TDX: <http://hdl.handle.net/10803/320188>

Doctorand/a: Claudia María Anleu Hernández

Títol: Latinoamericanos saliendo adelante. Una mirada desde la resiliencia sobre las migraciones y la intervención social con inmigrantes.

Any: 01/07/2015 | Universitat: URV

Direcció: Puig Cruells, Carmina; Soronellas Masdeu, Montserrat

CCUC: http://www.tdx.cat/handle/10803/314180

TDX:http://hdl.handle.net/10803/314180 
Doctorand/a: Cayuela Sánchez, Salvador

Títol: Agricultura Murciana y modos de vida en el contexto europeo

Any: 29/05/2015 | Universitat: URV

Direcció: Comas d'Argemir, Dolors; Schriewer, Klaus

CCUC: <http $/ /$ ccuc.cbuc.cat/ record $=$ b6411218 S23*cat $>$

TDX: <http://hdl.handle.net/10803/306267>

Doctorand/a: Muñoz Garcia, Araceli

Títol: La veu dels nens: experiències dels escolars de primària al voltant de la salut

Any: 19/05/2015 | Universitat: URV

Direcció: Larrea Killinger, Cristina

CCUC: <http://ccuc,cbuc.cat/ record=b6617165 S23* cat >

TDX: <http://hdl.handle.net/10803/318802>

Doctorand/a: Rodríguez Suárez, Roberto Alexis

Títol: Gusto musical y espacio social juvenil. Prácticas musicales en

Barcelona y Trójmiasto.

Any: 18/9/2014 | Universitat: URV

Direcció: Romaní Alfonso, Oriol; Martínez Sanmartí, Roger

CCUC:

Doctorand/a: Fernández Ferreira, Enrique Eduardo

Títol: Seguridad Urbana. Una perspectiva global centrada en las personas Any: 8/9/2014 | Universitat: URV

Direcció: Pujadas Muñoz, Joan Josep; Jordà Fernández, Antoni M.

CCUC: <http://ccuc.cbuc.cat/ record=b6298186 S23*cat>

TDX: <http://hdl.handle.net/10803/285050>

Doctorand/a: Anna Ciannameo

Títol: La migrazione del Chagas: costruzione bio-medica e sociopolitica delle malattie tropicali dimenticate / La migración de Chagas: la construcción bio-médica y socio-política de las enfermedades infecciosas tropicales

Any: 28/05/2014 | Universitat: URV/Univ. Bologna

Direcció: Comelles Esteban, Josep M. ; Brigati, Roberto; Quaranta, Ivo

CCUC: <http://ccuc.cbuc,cat/record=b6191227 S23* cat >

TDX: <http://amsdottorato.unibo.it/6517/1/ciannameo_anna_tesi.pdf > 
Doctorand/a: Massó Guijarro, Paloma

Títol: Un aleph en El Callejón del Gato. Espacio, cuerpo y ritual: una antropología de los tratamientos de heroína para la adicción a opiáceos en la biopolítica de la reducción de daños

Any: 7/4/2014 | Universitat: URV

Direcció: Romaní Alfonso, Oriol; March Cerdà, Joan Carles; Buzzaqui

Echevarrieta, Adrián

CCUC: <http $: / /$ ccuc,cbuc,cat/record $=$ b6298191 S23* cat $>$

TDX: <http://hdl.handle.net/10803/285316>

Doctorand/a: Barceló Prats, Josep

Títol: Poder local, govern i assistència pública: el cas de Tarragona

Any: 14/2/2014 | Universitat: URV

Direcció: Comelles Esteban, Josep M. ; Martorell Poveda, Maria Antònia

CCUC: <http // ccuc.cbuc.cat/record=b5969088 S23*cat>

TDX: <http //hdl.handle.net/10803/290988>

Doctorand/a: da Silva Adami, Vítor Hugo

Títol: O pensamento coletivo Hare Krishna e seus modos de institucionalização: um estudo sobre comunidades globalizadas e identidades locais

Any: 30/1/2014 | Universitat: URV

Direcció: Vallverdú Vallverdú, Jaume

CCUC: <http://ccuc.cbuc.cat/record=b5909240 S23*cat>

TDX: <http://www.tdx.cat/handle/10803/132850>

Doctorand/a: Feijoo Cid, Maria

Títol: 'Mi sangre está enferma, yo no’’ narrativas del mundo sida y la gestión del tratamiento

Any: 28/11/2013 | Universitat: URV

Direcció: Digiacomo, Susan M.

CCUC: <http://ccuc,cbuc,cat/record $=$ b5886313 S23* cat $>$

TDX: <http://www.tdx.cat/handle/10803/130933> 
Doctorand/a: Esteve Blanch, Jaume

Títol: La dependencia del alcohol: una comparación crítica sobre los tratamientos de dicha dependencia desde modelos biomédicos y espiritualistas

Any: 14/11/2013 | Universitat: URV

Direcció: Romaní Alfonso, Oriol

CCUC: <http $/ /$ ccuc,cbuc,cat/record=b5870889 S23* cat >

TDX: <http://www.tdx.cat/handle/10803/128946>

Doctorand/a: Oliveira Santos, Maria Consuelo

Títol: La dimensión estética de la salud en el Candomblé Ilê Axé Ijexá

Orixá Olufon -Sur de Bahía, Brasil-

Any: 16/7/2013 | Universitat: URV

Direcció: Muela Ribera, Joan ; Vallverdú Vallverdú, Jaume

CCUC: $<$ https://www.educacion.gob.es/teseo/mostrarRef.

do? ref $=1069908>$

Doctorand/a: Romizi, Francesco

Títol: El dios en la maleta. Los caminos de la significación mítica de la realidad de los ecuatorianos católicos en Barcelona y New York

Any: 9/7/2013 | Universitat: URV

Direcció: Pujadas Muñoz, Joan Josep

CCUC: <http $\$ / /$ ccuc,cbuc.cat/record $=$ b5858155 S23* cat $>$

TDX: <http://hdl.handle.net/10803/126447>

Doctorand/a: Masana Bofarull, Lina

Títol: El temps del mal. L'experiència i la gestió de la cronicitat en adults

Any: 18/6/2013 | Universitat: URV

Direcció: Comelles, Josep M.; Digiacomo, Susan M.

CCUC: <http://ccuc.cbuc.cat/record=b5743788 S23*cat>

TDX: <http://hdl.handle.net/10803/119547>

Doctorand/a: Sirvent Ribalda, Enrique

Títol: Del cuerpo regional a la totalidad viviente: saber y prácticas

anatómicas de las ciencias de la salud en Barcelona 1960-2012

Any: 23/5/2013 | Universitat: URV

Direcció: Comelles, Josep M. ; Arrizabalaga, Jon

CCUC: < https://www.educacion.gob.es/teseo/mostrarRef.

do?ref $=1042440>$

TDX: <http://hdl.handle,net/10803/119536> 
Doctorand/a: Ledo García, Julita

Títol: Usuarias de la red y Tcas. Un análisis antropológico de los espacios, usos y narrativas virtuales.

Any: 21/3/2013 | Universitat: URV

Direcció: Gracia Arnaiz, Mabel

CCUC:

Doctorand/a: Marxen, Eva María

Títol: «La comunidad silenciosa». Migraciones filipinas y capital social en el Raval (Barcelona)

Any: 6/11/2012 | Universitat: URV

Direcció: Martínez Hernàez, Àngel; Moreras Palenzuela, Jordi

CCUC: <http://ccuc,cbuc,cat/ record=b5397879 S23*cat>

TDX: <http://hdl.handle.net/10803/96667>

Doctorand/a: Albizu Beristain, Jose Luis

Títol: De la sonrisa Mcdonald's a la sonrisa de corazón. Los trabajadores locales, sus conocimientos situados y usos en un programa hacia la salud incluyente (Guatemala)

Any: 2/11/2012 | Universitat: URV

Direcció: Digiacomo, Susan M.

CCUC: <https://www.educacion.gob.es/teseo/mostrarRef. do?ref $=1010817>$

TDX: <http //hdl.handle.net/10803/101530>

Doctorand/a: Sabán, Mario Javier

Títol: El misterio de la Creación y el Árbol de la Vida en la mística judía.

Una interpretación del Maasé Bereshit

Anyः 23/10/2012 | Universitat: URV

Direcció: Prat Carós, Joan

CCUC: <http $/ /$ ccuc.cbuc.cat/record=b5397862 S23*cat>

TDX: <http://hdl.handle.net/10803/96298> 